\title{
Translational-orientational coupling during passage of methane through the bottleneck in zeolite $A$
}

\author{
R. Chitra and A. V. Anil Kumar \\ Solid State and Structural Chemistry Unit, Indian Institute of Science, Bangalore-560012, India \\ S. Yashonath* \\ Solid State and Structural Chemistry Unit and Supercomputer Education and Research Centre, \\ Indian Institute of Science, Bangalore-560012, India
}

\begin{abstract}
Diffusion of a five-site model of methane within porous zeolite A has been investigated by molecular dynamics simulation using potential available in the literature. Equilibrium and dynamical quantities especially those relating to the orientational degrees of freedom of methane have been computed. Methane exhibits interesting orientational preference during intercage diffusion through the eight-membered window which is the rate determining step for diffusion. A predominance of $(2+2)$ orientation in place of $(1+3)$ orientation is seen suggesting a strong translational-orientational coupling. This suggests that orientational motion might strongly influence the translational diffusivity of methane.
\end{abstract}

There has been an increasing interest in confined fluids during the past 15 years. ${ }^{1-6}$ This is not surprising since in nature materials are often found confined within host materials. Molecules and ions within membranes, hydrocarbons within zeolites, gases trapped in organic and inorganic hosts, and inclusion complexes are a few instances of these. Experimental and theoretical investigations of monatomic and polyatomic systems have shown that the properties of such fluids when confined within the porous solids are different and are known to exhibit some surprises. ${ }^{7-9}$

In recent times there have been many investigations of hydrocarbons adsorbed within zeolites as these systems are of considerable importance in the petrochemical industry. ${ }^{10-12}$ Methane is the simplest member of the hydrocarbon series which is often represented by a simple one-site Lennard-Jones potential. In this study we report a study of methane in zeolite $\mathrm{A}$ in which we examine the validity of this approximation as well as look at the importance of the orientational motion in understanding the properties of methane. The coupling between the translational and orientational motion is investigated during the diffusion of methane within the zeolite void network.

Zeolites are aluminosilicates which form a threedimensional network of interconnected void spaces. Zeolite A crystallizes in $F m \overline{3} c$ space group with a unit cell composition of $\mathrm{Na}_{32} \mathrm{Ca}_{32} \mathrm{Si}_{96} \mathrm{Al}_{96} \mathrm{O}_{384}$. The sodium and calcium ions are the extra framework cations essential for maintaining the charge neutrality of the unit cell arising out of the substitution of Si by Al. The void network consists of large cages, known as $\alpha$-cages of approximately $11.5 \AA$ in diameter interconnected via 8-membered rings of about $4.5 \AA$ diameter.

*Also at Condensed Matter Theory Unit, Jawaharlal Nehru Center for Advanced Scientific Research.
Molecular dynamics simulations have been carried out in the microcanonical ensemble on a system of $2 \times 2 \times 2$ unit cells of zeolite $\mathrm{NaCaA}$. The simulation cell is cubic with an edge length of $49.11 \AA$. Simulations have been performed at a somewhat dilute concentration of $1 \mathrm{CH}_{4}$ per $\alpha$-cage with a total of 64 methane molecules. Integration of the equations of motion of only methanes have been performed while the zeolite atoms have been kept fixed during the whole simulation. Periodic boundary conditions were imposed. The Gear predictor-corrector algorithm was used to integrate the equations of motion of the molecular centers of mass as well as those of the quaternions. A time step of 1 femtosecond (fs) was found to lead to adequate conservation of energy of about 1 in $10^{4}$. Calculations have been carried out at a temperature of $150 \mathrm{~K}$. An equilibration run of 200 ps was followed by a production run of $1 \mathrm{~ns}$ duration. The results presented below are based on the data stored every 0.05 ps.

The intermolecular potential parameters between the methane and the zeolite were derived from the literature. ${ }^{13,14}$ Lorentz-Berthelot rules were used to compute the crossinteraction parameters. The potentials were of the (6-12) Lennard-Jones form,

$$
\phi(r)=4 \epsilon\left[\left(\frac{\sigma}{r}\right)^{12}-\left(\frac{\sigma}{r}\right)^{6}\right]
$$

A five-site model was used for methane. The methane molecules were assumed to interact only with the oxygen atoms of the zeolite framework. The $\mathrm{Si}$ and $\mathrm{Al}$ atoms in the zeolite host are largely shielded by the surrounding oxygens, thus making the short-range interaction of these with the guest molecules insignificant. Table I lists the potential parameters for the methane-methane and methane-zeolite interactions.

Table II lists the average methane-methane, methanezeolite interactions, the total potential energy, as well as the 
TABLE I. Potential parameters for $\mathrm{CH}_{4}$ and $\mathrm{CH}_{4}-\mathrm{NaCaA}$ interactions.

\begin{tabular}{lll}
\hline \hline Type & $\sigma(\AA)$ & $\epsilon(\mathrm{kJ} / \mathrm{mol})$ \\
\hline $\mathrm{C}-\mathrm{C}$ & 3.350 & 0.405411 \\
$\mathrm{H}-\mathrm{H}$ & 2.813 & 0.068344 \\
& & \\
$\mathrm{C}-\mathrm{O}$ & 2.950 & 0.722931 \\
$\mathrm{C}-\mathrm{Na}$ & 3.360 & 0.126120 \\
$\mathrm{C}-\mathrm{Ca}$ & 3.350 & 1.967147 \\
$\mathrm{H}-\mathrm{O}$ & 2.682 & 0.296824 \\
$\mathrm{H}-\mathrm{Na}$ & 3.092 & 0.051783 \\
$\mathrm{H}-\mathrm{Ca}$ & 3.082 & 0.807680 \\
\hline \hline
\end{tabular}

average temperatures. The diffusion coefficient, $D$ is also listed in Table II. It is evident that at the dilute concentration of $1 \mathrm{CH}_{4}$ molecule per cage, the most significant contribution to the total interaction energy is from the methanezeolite interaction. The value obtained here $(\approx-15 \mathrm{~kJ} / \mathrm{mol})$ is slightly higher than that reported in the literature $(-18$ $\mathrm{kJ} / \mathrm{mol}){ }^{15}$ The diffusivities obtained here $\left(\approx 10^{-8} \mathrm{~m}^{2} / \mathrm{sec}\right)$ are somewhat higher than those obtained by Karger et al., ${ }^{16}$ based on PFG-NMR (Pulsed Field Gradient-NMR) measurements $\left(\approx 10^{-9}\right)$. However, when we note that these were for an A-type zeolite with a different $\mathrm{Si} / \mathrm{Al}$ ratio the difference seems acceptable. Demontis and Suffritti $^{17}$ have reported simulation of methane in NaA. They obtained differing values for $D$ depending on the precise parameter set employed by them. In view of these uncertainities and lack of experimental data it is difficult to fine-tune the parameters. We therefore make no claim about the accuracy of the chosen potential for the system under consideration viz. methane-NaCaA. However, since the focus of the present work is on translational-orientational coupling the purpose is served which is to illustrate the possibility of existence of such coupling.

As mentioned earlier the interconnecting eight-ring window is of rather narrow dimension $(4.5 \AA)$. This window is, therefore, the bottleneck for diffusion of molecules through the void network provided by the framework structure of zeolite A. Earlier diffusion of rare gases such as argon through zeolite A have been investigated. ${ }^{14}$ This study has shown that the sorbate-zeolite interaction plays a major role and the geometric factors such as the size and the shape of the diffusing species - which normally play the predominant role-does not do so in the case of argon in A. In the argonzeolite A study, the argon was a monatomic spherical par-

TABLE II. Average properties of $\mathrm{CH}_{4}$ in $\mathrm{NaCaA}$ from molecular dynamics run.

\begin{tabular}{ll}
\hline \hline Average property & $\mathrm{CH}_{4}$ in $\mathrm{NaCaA}$ \\
\hline$\left\langle T_{\text {trans }}\right\rangle(\mathrm{K})$ & 149.61 \\
$\left\langle T_{\text {rot }}\right\rangle(\mathrm{K})$ & 149.69 \\
$\left\langle U_{g g}\right\rangle(\mathrm{kJ} / \mathrm{mol})$ & -0.335190 \\
$\left\langle U_{g h}\right\rangle(\mathrm{kJ} / \mathrm{mol})$ & -14.759575 \\
$\left\langle U_{\text {tot }}\right\rangle(\mathrm{kJ} / \mathrm{mol})$ & -15.093094 \\
$D_{\text {trans }}\left(\mathrm{m}^{2} / \mathrm{sec}\right)$ & $2.4999 \times 10^{-8}$ \\
$D_{\text {rot }}(/ \mathrm{sec})$ & $2.61 \times 10^{13}$ \\
\hline \hline
\end{tabular}

ticle and as a consequence there were no orientational degrees of freedom. In this study, we would like to look at the role of orientational motion on the properties especially of the diffusion of methane within zeolite A.

We focus on intercage diffusion event, since this is the rate determining step contributing to long-time diffusion. Each methane molecule is said to belong to that $\alpha$-cage whose center is closest to the molecular center of mass. When a molecule belongs to different cages at successive steps, a cage-to-cage crossover is said to have occurred. The temperature of the simulation is $150 \mathrm{~K}$. There were 1193 cage-to-cage crossovers during the $1 \mathrm{~ns}$ simulation. The variation of the methane-zeolite interaction energy as a function of perpendicular distance, $d$, from the window plane, indicates that there is no energetic barrier for the diffusion of methane across the eight-ring window. This is in accordance with earlier findings that the barrier at the window depends on the levitation parameter, ${ }^{18}$

$$
\gamma=\frac{2.2^{1 / 6} \cdot \sigma_{\mathrm{sz}}}{\sigma_{w}},
$$

and this barrier is negative for $\gamma \approx 1$. In the present case the size of the methane molecule is comparable to that of the eight-ring window and consequently, $\gamma$ is close to unity. The energetic barrier is, therefore, negative.

The question of interest is whether methane has any preferential orientation within (i) the cage and (ii) during its passage through the window. Our analysis of the MD trajectories showed no significant preference for any particular orientation of methane within the cage itself. On the contrary, the analysis indicated preference for certain orientations during its passage through the window. The method of analysis of the orientation is outlined below.

The orientation of methane can be characterized by the orientation of the $\mathrm{C}-\mathrm{H}$ bond with respect to the vector perpendicular to the window plane, $\hat{\mathbf{n}}$. The dot product of each of the $\mathrm{C}-\mathrm{H}$ bonds is calculated with $\hat{\mathbf{n}}$. The magnitude of the largest of the four dot products is used to obtain $\theta$. The tetrahedral symmetry of the molecule limits the range of variation of $\theta$ between 0 and $\theta_{T_{d}}$, where $\theta_{T_{d}}$ is the tetrahedral angle. Two ideal orientations may be considered: (i) where one of the $\mathrm{C}-\mathrm{H}$ bond vectors is perpendicular to the window plane, so that the other three $\mathrm{C}-\mathrm{H}$ bonds are spread like an inverted umbrella. We refer to this orientation as $(3+1)$, and (ii) the other orientation in which two of the $\mathrm{C}-\mathrm{H}$ bonds are symmetrical with respect to $\hat{\mathbf{n}}$ and make an angle of $\theta_{T_{d}} / 2$ with $\hat{\mathbf{n}}$. This is referred to as $(2+2)$ orientation. A range of orientations between these two idealized $(3+1)$ and $(2+2)$ orientations are possible.

A simple analysis can be carried out as follows: each molecule is assigned to one of these two standard orientations, based on whether the angle $\theta$ lies in the interval $(0$, $\left.\theta_{T_{d}} / 4\right)[(3+1)$ orientation $]$ or $\left(\theta_{T_{d}} / 4, \theta_{T_{d}} / 2\right)[(2+2)$ orientation]. Figure 1 shows a plot of the percentage of molecules which are in any given orientation as a function of the distance from the window plane, $d$. Note that when $d=0$, the molecular center-of-mass is exactly in the window plane. It can be seen that when the molecule is far away from the window plane and deep within the $\alpha$-cage, there is no notice- 


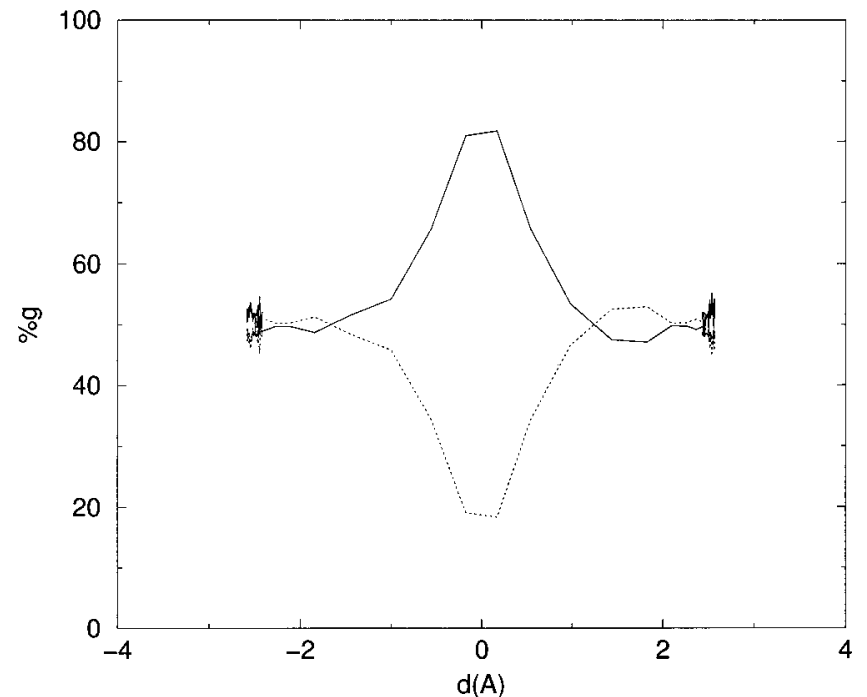

FIG. 1. Percentage of methanes, $\% \mathrm{~g}$ with $(2+2)$ and $(3+1)$ orientation as a function of the perpendicular distance of the methane center-of-mass from the plane of the eight-membered ring window through which it passes during intercage migration. Negative values of $d$ (in Angstroms) indicate that the methane is in the parent cage and positive values in the neighboring daughter cage. Note the strong preference for the $(2+2)$ orientation in the vicinity of the eight-ring window in zeolite $\mathrm{NaCaA}$.

able preference for either the $(3+1)$ or the $(2+2)$ orientation, the error in the estimated value being $2 \%-3 \%$. But when the molecule is in the window plane, there exists a strong preference for $(2+2)$ orientation $(78 \%)$. Figure 2 shows a snapshot of a single methane molecule at equispaced MD time steps just before, during, and subsequent to an intercage crossover event. It is evident that the molecule is at $(2+2)$ orientation just when it is at the window plane.

While Fig. 1 gives an idea of the number of molecules which prefer to align into the $(2+2)$ orientation, it does not indicate the degree with which this alignment occurs. In order to quantify the degree of alignment, we define

$$
\zeta=\frac{\theta}{\theta_{T_{d}} / 2},
$$

which behaves like an order parameter $[0$ for the standard $(3+1)$ and 1 for standard $(2+2)$ orientation]. However, our earlier definition would classify all values between 0 and 0.5
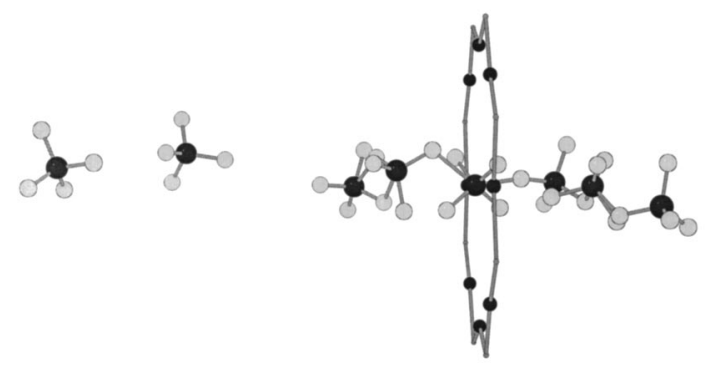

FIG. 2. A series of snapshots showing the orientation of methane just before, during, and soon after its passage through the eight-ring window in zeolite $\mathrm{NaCaA}$. Note the assuming of the $(2+2)$ orientation of the methane molecule just before its passage through the eight-ring window as also the presence of other orientations when methane is far away from the window plane.
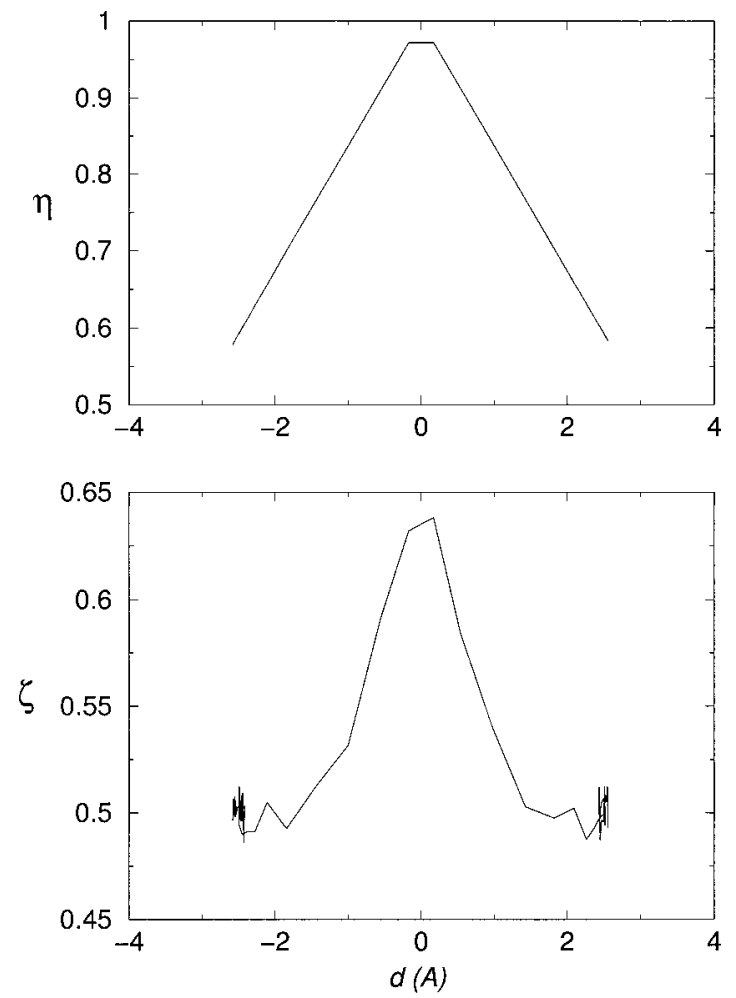

FIG. 3. Variation of $\zeta$ and $\eta$ (see text) during the intercage diffusion event of a methane obtained by averaging over all such events. Note the strong preference for $(2+2)$ as suggested by the value of 0.64 for $\zeta$ at $d=0 \AA$.

as $(3+1)$ orientations, while those between 0.5 and 1 would be considered as $(2+2)$ orientations. A plot of $\zeta$ is shown against $d$ in Fig. 3. It is seen that $\zeta \sim 0.5$ when the molecule is far away from the window plane indicating an average orientation that lies midway between $(3+1)$ and $(2+2)$ orientations. This is not the case, however, near the window plane where $\zeta$ increases to 0.63 or 0.64 at $150 \mathrm{~K}$, suggesting an orientation closer to the $(2+2)$ orientation and away from the $(3+1)$ orientation.

A translational order parameter, $\eta$ can be defined as follows:

$$
\eta=\frac{(a / 4)-d_{w}}{(a / 4)}
$$

where $a$ is the unit cell parameter $24.555 \AA$ and $d_{w}$ is the perpendicular distance of the center-of-mass from the window plane. $\eta$ equals 0 if the molecule is at any $\alpha$-cage center and is 1 when it is at the window plane. The variation of $\eta$ is also shown in Fig. 3. The correlation coefficient $r$, between the translational order parameter $\eta$ and the orientational order parameter $\zeta$ is defined as

$$
r=\frac{\sum_{i=1}^{n} \eta \zeta-n \bar{\eta} \bar{\zeta}}{n \sigma_{\eta} \sigma_{\zeta}},
$$

where $\bar{\eta}$ and $\bar{\zeta}$ indicate mean values, while $\sigma_{\eta}$ and $\sigma_{\zeta}$ denote the standard deviations in the two quantities. Here $n$ is the number of intercage crossover events (1193 in the present case). The correlation coefficient $r$ is a measure of the translational-orientational coupling. It is found that $r$ gives a value of 0.5145 when the data over the $(-2,2)$ ps of the 
cage-to-cage crossover event is included in the calculation of $r$ and 0.9285 when only the data over $(-0.4,0.4) \mathrm{ps}$ is included. The latter ensures that only data in the vicinity of the window plane are included. The higher value of $r$ suggests that there might be a strong translational-orientational coupling during cage-to-cage migration. It is possible to plot $r$ as a function of $d$ if a longer simulation is carried out and this would show that $r$ is largest when $d \rightarrow 0$.

In conclusion, it is found that the methane molecule exhibits a strong orientational preference during its passage through the bottleneck which is an eight-membered ring. As the passage through this rather narrow window is the rate determining step for diffusion within the zeolite, such orientational preference could be of considerable importance. An estimate of $r(0.93)$ suggests strong translationalorientational coupling during the passage through the eightring window. This finding is likely to be of considerable importance in understanding diffusion of not only methane but also other higher hydrocarbons within zeolitic voids. The importance of hydrocarbon-zeolite system in petrochemical industries cannot be overemphasized. The possibility of hydrogen abstraction from the methane by the oxygen of the zeolite framework could be affected by the rate of reorientation of methane: the slower the reorientational motion, the higher the probability that hydrogen will spend sufficiently long time near a particular oxygen atom which could increase the possibility of abstraction of hydrogen from the methane. We found that the methane reorientational motion slows down during its passage through the window although the translational displacement is accelerated. In the long term, such knowledge is likely to be useful in designing better adsorbents with higher separation factors and lower energy cost.

It is to be expected that orientational preference for a particular orientation should decrease with increase in temperature. As the kinetic energy increases and when $k T$ $\gg \Delta u$ where $\Delta u=u(2+2)-u(1+3)$ is the difference between the $(2+2)$ and $(1+3)$ orientations, the orientational preference will be negligible. However, in systems where $\Delta u$ is very large then such a situation may not be attainable at any realistic temperature. This can happen, for example, when the molecule is highly anisotropic and therefore it is able to pass through the window only when it is in a particular orientation out of the many possible orientations.

The authors gratefully acknowledge support from the Department of Science \& Technology, New Delhi under a project entitled Investigations into Diffusion of Polyatomic Molecules through Porous Media.

${ }^{1}$ J. Karger and D. M. Ruthven, in Diffusion in Zeolites and Other Microporous Solids (Wiley, New York, 1992).

${ }^{2}$ S. P. Bates and R. A. VanSanten, Adv. Catal. 42, 1 (1997).

${ }^{3}$ J. Valyon, Gy. Onyestyk, and L. V. C. Rees, Langmuir 16, 1331 (2000).

${ }^{4}$ R. L. June, A. T. Bell, and D. N. Theodorou, J. Phys. Chem. 95, 8866 (1991).

${ }^{5}$ Complete issue of J. Chem. Soc., Faraday Trans. 2 87(13), 1947-2119 (1991).

${ }^{6}$ G. B. Woods and J. S. Rowlinson, J. Chem. Soc., Faraday Trans. 2 85, 765 (1989).

${ }^{7}$ K. Hahn and J. Kärger, J. Phys. Chem. 100, 316 (1996).

${ }^{8}$ E. G. Derouane, J-M. Andre, and A. A. Lucas, J. Catal. 110, 58 (1988).

${ }^{9}$ C. Saravanan, F. Jousse, and S. M. Auerbach, Phys. Rev. Lett. 80, 5754 (1998).

${ }^{10}$ R. Q. Snurr, J. Phys. Chem. 102, 6720 (1998).

${ }^{11}$ L. N. Gergidis, D. N. Theodorou, and H. Jobic, J. Phys. Chem. B 104, 5541 (2000).

${ }^{12}$ F. Marquez, H. Garcia, E. Palomares, L. Fernandez, and A. Corma, J. Am. Chem. Soc. 122, 6520 (2000).

${ }^{13}$ S. Murad and K. E. Gubbins, ACS Symp. Ser. 86, 62 (1978).

${ }^{14}$ P. Santikary and S. Yashonath, J. Chem. Soc., Faraday Trans. 88, 1063 (1992).

${ }^{15}$ H. Yucei and D. M. Ruthven, J. Chem. Soc., Faraday Trans. 76, 60 (1980).

${ }^{16}$ J. Karger, H. Pfeifer, M. Rosemann, N. N. Flokistova, and S. P. Zdanov, Zeolites 9, 247 (1989).

${ }^{17}$ P. Demontis and G. B. Suffritti, Mol. Phys. 91, 669 (1997).

${ }^{18}$ S. Yashonath and P. Santikary, J. Phys. Chem. 98, 6368 (1994). 